\title{
Evaluation of the True Wavefront Aberrations in Eyes Implanted With a Rotationally Asymmetric Multifocal Intraocular Lens
}

\author{
Vyas Akondi, PhD; Pablo Pérez-Merino, PhD; Eduardo Martinez-Enriquez, PhD; Carlos Dorronsoro, PhD; \\ Nicolás Alejandre, MD; Ignacio Jiménez-Alfaro, MD, PhD; Susana Marcos, PhD
}

\begin{abstract}
PURPOSE: Standard evaluation of aberrations from wavefront slope measurements in patients implanted with a rotationally asymmetric multifocal intraocular lens (IOL), the Lentis Mplus (Oculentis $\mathrm{GmbH}$, Berlin, Germany), results in large magnitude primary vertical coma, which is attributed to the intrinsic IOL design. The new proposed method analyzes aberrometry data, allowing disentangling the IOL power pupillary distribution from the true higher order aberrations of the eye.
\end{abstract}

METHODS: The new method of wavefront reconstruction uses retinal spots obtained at both the near and far foci. The method was tested using ray tracing optical simulations in a computer eye model virtually implanted with the Lentis Mplus IOL, with a generic cornea or with anterior segment geometry obtained from custom quantitative spectral-domain optical coherence tomography in a real patient. The method was applied to laser ray tracing aberrometry data at near and far fixation obtained in a patient implanted with the Lentis Mplus IOL.

RESULTS: Higher order aberrations evaluated from simulated and real retinal spot diagrams following the new reconstruction approach matched the nominal aberrations (approximately 98\%). Previously reported primary vertical coma in patients implanted with this IOL lost significance with the application of the proposed reconstruction.

CONCLUSIONS: Custom analysis of ray tracing-based retinal spot diagrams allowed decoupling of the true higher order aberrations of the patient's eye from the power pupillary distribution of a rotationally asymmetric multifocal IOL, therefore providing the appropriate phase map to accurately evaluate through-focus optical quality.

[J Refract Surg. 2017;33(4):257-265.]

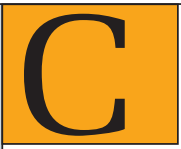

ataract is successfully treated by replacement of the crystalline lens with an intraocular lens (IOL). Monofocal IOLs are preferably set for far vision and reading glasses are used for near vision. The dependency on glasses after cataract surgery can be eliminated with the help of multifocal IOLs that provide multiple foci and thereby enable functional simultaneous vision at far, intermediate, and near distances. Most designs of multifocal IOLs are diffractive, refractive, or hybrid. ${ }^{1}$

A rotationally asymmetric refractive multifocal IOL design has gained popularity in the past decade. This design provides two distinct foci with its two definite correcting zones for far and near vision. In general, there is a smooth transition between the two zones. The Lentis Mplus IOL (Oculentis GmbH, Berlin, Germany) is based on this design and has demonstrated good near and distance visual outcomes and good contrast sensitivity. ${ }^{2}$ The presence of a large magnitude intraocular primary coma has been reported in patients implanted with this multifocal IOL, which is usually attributed to its vertical asymmetric optical geometry. ${ }^{3,4}$ In addition, it is believed that the presence of this optical defect allows an extended depth of focus that would grant adequate vision at various distances. On the other hand, it is assumed that this additional coma causes

From Visual Optics and Biophotonics Laboratory, Instituto de Óptica "Daza de Valdés," Madrid, Spain; and Fundación Jiménez Díaz, Madrid, Spain.

Submitted: August 10, 2016; Accepted: November 9, 2016

(C) 2017 Akondi, Pérez-Merino, Martínez-Enriquez, et al.; licensee SLACK Incorporated. This is an Open Access article distributed under the terms of the Creative Commons Attribution 4.0 International (https://creativecommons. org/licenses/by/4.0). This license allows users to copy and distribute, to remix, transform, and build upon the article, for any purpose, even commercially, provided the author is attributed and is not represented as endorsing the use made of the work.

Supported by Consejería de Educación, Juventud y Deporte of Comunidad de Madrid and the People Programme (Marie Curie Actions) of the European Union's Seventh Framework Programme (FP7/2007-2013) under REA grant agreement no. 291820 to VA; ERC Grant Agreement [ERC-2011-AdG 294099] and Spanish Government Grants FIS2011-25637 and FIS2014-56643-R to SM.

Correspondence: Vyas Akondi, PhD, C/Serrano 121, Visual Optics and Biophotonics Laboratory, Instituto de Óptica "Daza de Valdés," Madrid 28006, Spain.E-mail: vyas.akondi@io.cfmac.csic.es

doi:10.3928/1081597X-20161206-03 


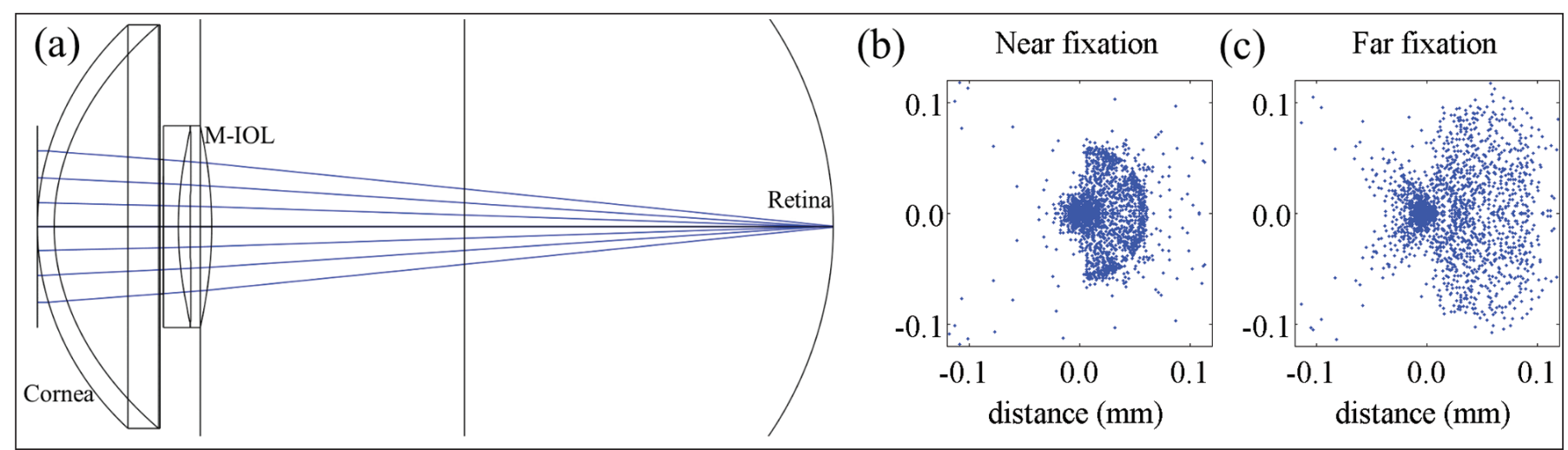

Figure 1. (A) Side view of the optical layout of a pseudophakic computer eye model with the Lentis Mplus intraocular lens (Oculentis GmbH, Berlin, Germany) accurately simulated by including the effects of the transition region; ray tracing spot diagrams (51 $\times 51$ sampling) obtained at (B) near focus and (C) far focus. M-IOL = multifocal intraocular lens

blur and limits vision outcomes in certain cases, which was previously attributed to the imbalance between the blur induced by coma and the visual benefit from it.

We show that most commercially available wavefront sensors using standard wavefront reconstruction methods do not allow an accurate measurement of the total wavefront aberrations (true ocular aberrations + power pupillary distribution due to the multifocal IOL) in patients implanted with segmented rotationally asymmetric multifocal IOLs such as the Lentis Mplus IOL. Hence, it is inappropriate to conclude that this IOL induces vertical coma by performing aberrometry analysis with these instruments. It is worth noting that conventional aberrometry also fails in diffractive multifocal IOLs, as argued earlier. ${ }^{5}$

We report a novel method of separating the aberrations of the patient's eye from the rotationally asymmetric multifocal IOL power distribution across the pupil. The proposed wavefront reconstruction method involves appropriately combining wavefront slopes estimated at far and near distances. The method is tested through ray tracing simulations with prediction of aberrations from an optical coherence tomography-based customized computer eye model. The proposed wavefront reconstruction methodology is applied to wavefront slope measurements made by a laser ray tracing (LRT) aberrometer at far and near fixating conditions. It is shown that a significantly large magnitude vertical coma, which was detected in previous studies, is a result of the application of conventional aberrometric analysis that is not suitable to recover aberrations in patients implanted with a segmented multifocal IOL. The separation of the patient's ocular aberrations from the IOL power pupillary pattern using the proposed method allows a true estimate of the through-focus retinal image quality, which can be used to predict the optimal orientation of the IOL. ${ }^{6}$

\section{PATIENTS AND METHODS NEW METHOD FOR WAVEFRONT RECONSTRUCTION}

The Hartmann-Shack wavefront sensor ${ }^{7}$ and LRT aberrometry $^{8}$ share the same principle for wavefront reconstruction, by which local wavefront slopes are estimated by measuring the location of the focal spots. In LRT, local slopes are obtained by sequentially scanning a laser beam at different predetermined pupil locations and recording the focal spots corresponding to each pupil location, whereas the Hartmann-Shack wavefront sensor measures all local wavefront slopes at the same time with the help of a microlens array. ${ }^{9,10}$ In either case, the evaluation of local wavefront slopes involves estimation of the location of the spots using centroid detection methods at a fixed plane of focus (generally at best focus).

The near and far correcting sectors of the Lentis Mplus IOL focus at two different locations. ${ }^{2}$ While examining patients implanted with a rotationally asymmetric multifocal IOL such as the Lentis Mplus IOL using a standard commercial Hartmann-Shack wavefront sensor, it is not possible to simultaneously obtain sharp focal spot images from near and far correcting regions of the multifocal IOL. This is due to the fact that the spots arising from the near correcting portion of the IOL are not well focused when measurements are performed with far fixation because these focal spots are affected by the multifocal IOL power distribution in the near correcting portion. Similarly, with near fixation, the spots obtained from the far correcting portion of the IOL are not focused.

Figure 1A shows the side view of the optical layout of a simple computer eye model ${ }^{11}$ in which the crystalline lens is replaced by the Lentis Mplus IOL, simulated in an optical design program (Zemax, LLC, Redmond, WA). The IOL is accurately simulated by including the effects of the transition region. 
Through-focus visual Strehl ratio, computed from the Optical Transfer Function using the phase due to the IOL (extracted using Zemax software) showed that the IOL under examination has a near distance addition of +3.00 diopters (D). This is also confirmed with through-focus evaluation based on other metrics, including peak irradiance, modulation transfer function at 50 cycles/degree, and area under the curve modulation transfer function. Numerical ray tracing was performed on-axis for near and far fixation with the help of a custom developed Zemax macro to obtain the locations of the traced rays at the retina and retinal spot diagrams $(51 \times 51$ rectangular sampling $)$ for near (Figure 1B) and far (Figure 1C) distances.

Standard reconstruction methods use slope measurements made at a single plane (with near, intermediate, or far fixation), assuming a spherical reference wavefront with a single point of focus, to obtain the wavefronts. The new proposed method of wavefront reconstruction, called the multifocal reconstruction method, combines the true local wavefront slopes measured using the genuine focal spots obtained at near and far fixation. The proposed method is applicable in patients with segmented multifocal IOL implants. It separates the true ocular aberrations from the multifocal IOL power pupillary distribution. The following sections describe the procedure to reconstruct the wavefront aberrations and to evaluate the multifocal IOL power pupillary distribution from the slope measurements.

\section{WAVEFRONT RECONSTRUCTION FrOM SLOPE MEASUREMENTS}

The nature of the proposed method requires measuring the location of the Hartmann-Shack or LRT focal spots at both near and far fixation. The wavefront aberrations can be reconstructed from the local wavefront slopes in two different ways: zonal or modal fitting. In zonal fitting, the wavefront is analytically derived over the whole surface using local wavefront slope information. Modal fitting is described in terms of smoothly varying orthogonal set of modes by fitting the wavefront slopes with the gradients of the modes. Although both approaches produced the same result when genuine focal spots are used in the proposed reconstruction method, for the purpose of demonstrating the abrupt changes that occur near the transition region while using the standard reconstruction method, the zonal fitting approach was applied to both the standard and proposed wavefront reconstruction methods.

Wavefront reconstruction involves the estimation of local wavefront slopes from the ray tracing spot diagrams. The local ' $x$ ' and ' $y$ ' wavefront slopes were eval- uated by dividing the ' $x$ ' and ' $y$ ' coordinates of the centroid location of the spots with the focal length. Matrix formulation was used to relate the phase in terms of the measured local slope values using the slope geometry of Southwell. ${ }^{12}$ With the help of the singular value decomposition technique for matrix inversion, the wavefront was reconstructed..$^{13}$

In computer model eyes with nominal aberrations, the residual wavefront error was estimated by subtracting the nominal wavefront from the reconstructed wavefront. The Zernike coefficients, $a_{i}(0 \leq i \leq 35)$, obtained after decomposing the resultant residual wavefront error were used to evaluate the root mean square (RMS) wavefront error, which is given by the following expression:

$$
R M S=\sqrt{\sum_{i=3}^{i=35} a_{i}^{2} .}
$$

Piston and tilt terms were not included in the evaluation of the RMS wavefront error.

\section{Evaluation of the Multifocal IOL Power MaP}

The use of single plane slope measurements for wavefront reconstruction in a standard method is straightforward, as described above. The proposed multifocal reconstruction method requires combining the true wavefront slope measurements from near and far fixation. This was done with the help of a binary reference map ( 1 for far fixation and 0 for near fixation) that identifies the pupillary location of the true wavefront slopes from the slope values at the two distances (far and near). The binary reference map was obtained in an aberration-free pseudophakic computer eye model with the Lentis Mplus IOL by comparing the corresponding pairs of slope values at near and far distances and choosing the slope value closer to zero as the true slope value.

\section{WAVEFRONT RECONSTRUCTION SIMULATIONS IN A COMPUTER EYE MODEL}

The proposed multifocal reconstruction method was tested and compared with the standard reconstruction method using simulations on computer eye models:

1. Pseudophakic computer eye model with the Lentis Mplus IOL (two distinct focusing planes) and in the absence of aberrations.

2. Pseudophakic computer eye model with the Lentis Mplus IOL with different Zernike aberrations, including primary defocus, primary astigmatism, primary coma, and primary spherical aberrations induced at the pupil plane. The Zernike coefficient corresponding to each higher order aberration (HOA) is $0.275 \mu \mathrm{m}$. 
3. Pseudophakic computer eye model with the Lentis Mplus IOL and with statistically simulated aberrations generated using Zernike coefficient data of 41 patients obtained from the literature. ${ }^{14}$ Here, piston and tilt terms that do not distort the point spread function of the optical system were not included in generating the wavefronts.

4. Customized computer eye model based on threedimensional OCT measurements on a patient implanted with the Lentis Mplus IOL.

All simulations were performed with a 4-mm pupil diameter.

\section{Patient ANd SuRgery}

The case study involved retrospective analysis of one patient (age: 60 years; preoperative refraction: $+1.75 \mathrm{D}$ ) implanted with a Lentis Mplus IOL (Model: LS-312; IOL power: $+21.50 \mathrm{D}$; with IOL power calculated using the SRK-T formula, selecting the first myopic value closest value to emmetropia). The surgery was performed by one of the authors (IJ-A) at Fundación Jiménez Díaz using a 2.2-mm incision at $90^{\circ}$. A $6-\mathrm{mm}$ continuous curvilinear capsulorhexis was made under viscoelastic material. Phacoemulsification of the lens was performed with a commercial microsurgical system (Stellaris Microsurgical System; Bausch \& Lomb, Rochester, NY). The postoperative refraction was: $-0.25-0.75 \times 105^{\circ}$; corrected distance visual acuity was $20 / 20$ and corrected near visual acuity was 20/25. Consent was obtained from the patient and the procedure was explained in detail. The study met the tenets of the Declaration of Helsinki and the protocols were approved by the institutional review boards of CSIC and Fundación Jiménez Díaz.

\section{Quantitative ANTERIOR Segment OCT}

A custom-developed quantitative spectral-domain OCT (840 nm, 50-nm bandwidth) was used to measure the three-dimensional geometry of the anterior segment of the patient's eye. ${ }^{15}$ A-scans were acquired at a speed of 25,000 Hz. Five sets of three-dimensional images (raster scanning of 350/50 A-scans/B-scans, $10 \mathrm{~mm}$ lateral range) were focused at the corneal plane. The patient fixated on a stimulus that was moved in a minidisplay conjugate to the retina (focused with a Badal system) to align the keratometric axis with the OCT axis and allowed estimation of the eccentric position of the fovea. Fully automated custom developed image processing tools for distortion correction, denoising, segmentation, and surface fitting were used to quantify the anterior and posterior surfaces of the cornea of the patient. ${ }^{15-19}$ The OCT predictions of corneal geometry have been earlier validated with ex vivo artificial model eyes using non-contact profilometry and in vivo comparison with Scheimpflug and videokeratography. ${ }^{17-19}$

\section{OCt-BAsed Customized Computer Eye Model}

The surface profile heights of the anterior and posterior corneal surfaces measured with the anterior segment OCT were used to construct a customized computer eye model in Zemax based on the patient anatomical measurements. The nominal IOL geometry was introduced in Zemax in the three-dimensional arrangement obtained from OCT. Retinal spot diagrams obtained in the customized computer eye model were used for wavefront estimation.

\section{LRT MEASUREMENTS}

LRT measurements were obtained on a customdeveloped system using a hexagonal sampling geometry, which improved sampling efficiency on a circular aperture. ${ }^{20}$ Slope measurements were obtained at 91 pupil sampling locations (5-mm pupil diameter). Each LRT spot image on a different frame of a CCD camera was captured using $255 \times 255$ pixels $(7.4-\mu \mathrm{m}$ pixel size). The total time taken to capture all 91 spots was 3 seconds. The patient's pupil was continuously monitored with a CCD camera in a pupil monitoring channel. The patient was stabilized using a bite bar.

The standard reconstruction method and proposed multifocal reconstruction method were applied on LRT slope measurements obtained with a hexagonal sampling geometry. Nearest-neighbor interpolation, which preserves the abrupt changes in local slope values, was performed to reduce the hexagonal sampling geometry to a rectangular sampling. The potential wavefront error induced by the applied interpolation method was tested with a simple computer eye model and it was found that the RMS wavefront error in wavefront reconstruction due to interpolation was lower than $0.02 \mu \mathrm{m}$ when primary defocus, primary $45^{\circ}$ astigmatism, and primary vertical coma were induced separately, each with a corresponding Zernike coefficient of $0.25 \mu \mathrm{m}$.

\section{RESULTS}

\section{WAVEFront Reconstruction in a PSEUdophakic Eye MODEL}

Wavefront aberrations were obtained for a simple eye model virtually implanted with the Lentis Mplus IOL, using the standard reconstruction method and the proposed multifocal reconstruction method.

Figure A (available in the online version of this article) shows the local ' $x$ ' and ' $y$ ' slopes of the detected wavefront and the reconstructed wavefront using the standard method in near, far, and intermediate fixation in the "Lentis-implanted" computer model eye. A 


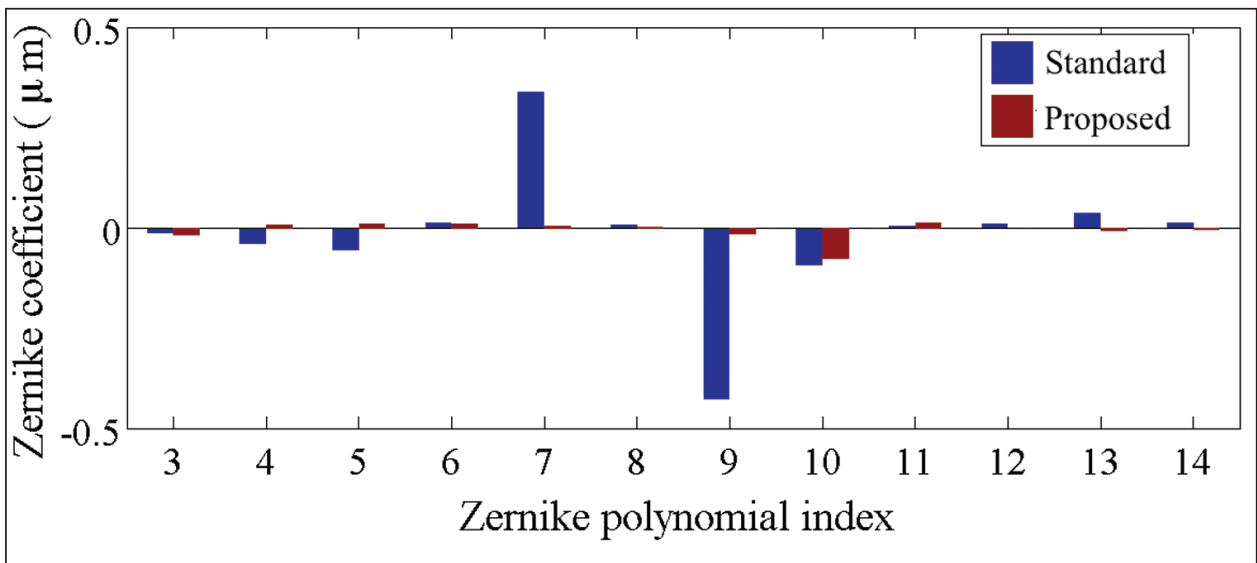

Figure 2. Comparison of the reconstructed higher order Zernike coefficients (pseudophakic computer eye model with the Lentis Mplus intraocular lens [Oculentis $\mathrm{GmbH}$, Berlin, Germany] and with no aberrations) using the standard reconstruction method in a single (intermediate) plane of focus (blue bars) and the proposed multifocal reconstruction method (brown bars) that combines the slope values from near and far wavefront sensor measurements. Data are for 4-mm pupil diameter.

phase discontinuity near the transition region is evident from these local slope maps. The standard reconstruction method (ie, using local slopes from a single plane of focus) resulted in high levels of aberrations. The wavefronts reconstructed with the standard method resulted in a partial defocus for near (positive primary Zernike defocus coefficient) and far (negative primary Zernike defocus coefficient) distance cases and primary vertical coma (resulting from a change in the sign of defocus in far and near correcting regions of the IOL) in the intermediate distance. The RMS wavefront error of the reconstructed aberration is $1.56 \mu \mathrm{m}$ at near fixation and $1.64 \mu \mathrm{m}$ at far fixation (for $4-\mathrm{mm}$ pupils).

The wavefront obtained using the proposed multifocal reconstruction method is shown in Figure AD. The HOA component is much flatter because the method separates out the pupillary power distribution of the multifocal IOL, which is shown in Figure AE. The RMS wavefront error of the HOA component obtained with the proposed multifocal reconstruction method is $0.10 \mu \mathrm{m}$ (with a dominant spherical aberration of magnitude $0.08 \mu \mathrm{m}$ ) and the peak-to-valley is $0.31 \mu \mathrm{m}$ (for 4-mm pupils).

The reconstructed wavefronts obtained in the standard and proposed methods were decomposed into the first seven orders of Zernike polynomials (36 Zernike coefficients) using the least-square fitting technique and the decomposed wavefronts are shown in Figure A. Figure 2 compares the first four orders of Zernike polynomial coefficients for wavefronts obtained with the standard reconstruction method (intermediate fixation) and the proposed multifocal reconstruction method. The Zernike coefficients resulting from the application of the proposed multifocal reconstruction method are closer to zero, whereas the standard reconstruction method results in significantly large magnitudes of vertical coma $(0.34 \mu \mathrm{m})$ and a trefoil term $(-0.43 \mu \mathrm{m})$.

A comparison of the nominal eye's wave aberrations and the wave aberration reconstructed using the pro- posed multifocal reconstruction method in simulated aberrated pseudophakic eyes implanted with the Lentis Mplus IOL is shown in Figure B (available in the online version of this article). The estimated residual wavefronts (Figure B) while sensing the induced primary $45^{\circ}$ astigmatism, vertical primary coma, and primary spherical aberrations had RMS wavefront errors of $0.09,0.10$, and $0.11 \mu \mathrm{m}$, respectively.

The proposed multifocal reconstruction method was also applied on nine pseudophakic computer eye models implanted with the Lentis Mplus IOL. In each case, a statistically estimated aberration is simulated..$^{14}$ The average RMS wavefront error (from the nominal values) was $0.11 \pm 0.02 \mu \mathrm{m}$, demonstrating the potential of the proposed multifocal reconstruction approach in reconstructing the wavefront aberration in realistic eyes. On average, the difference of the vertical coma Zernike coefficients obtained using the standard reconstruction method on a single plane of focus at an intermediate fixation and the proposed multifocal reconstruction approach was $0.33 \pm 0.02 \mu \mathrm{m}$.

\section{WAVEFRONT RECONSTRUCTION IN A CUSTOMIZEd COMPUTER EYE MODEL}

Figure CA (available in the online version of this article) illustrates a two-dimensional cross-section of the recorded three-dimensional OCT image, the anterior segment biometric parameters evaluated from a fully automated image processing method, and the aberration map calculated from the estimated corneal topography. Figures CB-CD show the local ' $x$ ' slopes, ' $y$ ' slopes, and the reconstructed wavefront at far, near, and intermediate fixation from ray tracing in a customized eye model using this topographic information. Figure CE shows the wavefront resulting from the combination of the near and far fixation measurements based on the proposed multifocal reconstruction method. The wavefront obtained by using the proposed multifocal reconstruction method (Figure CE) closely 


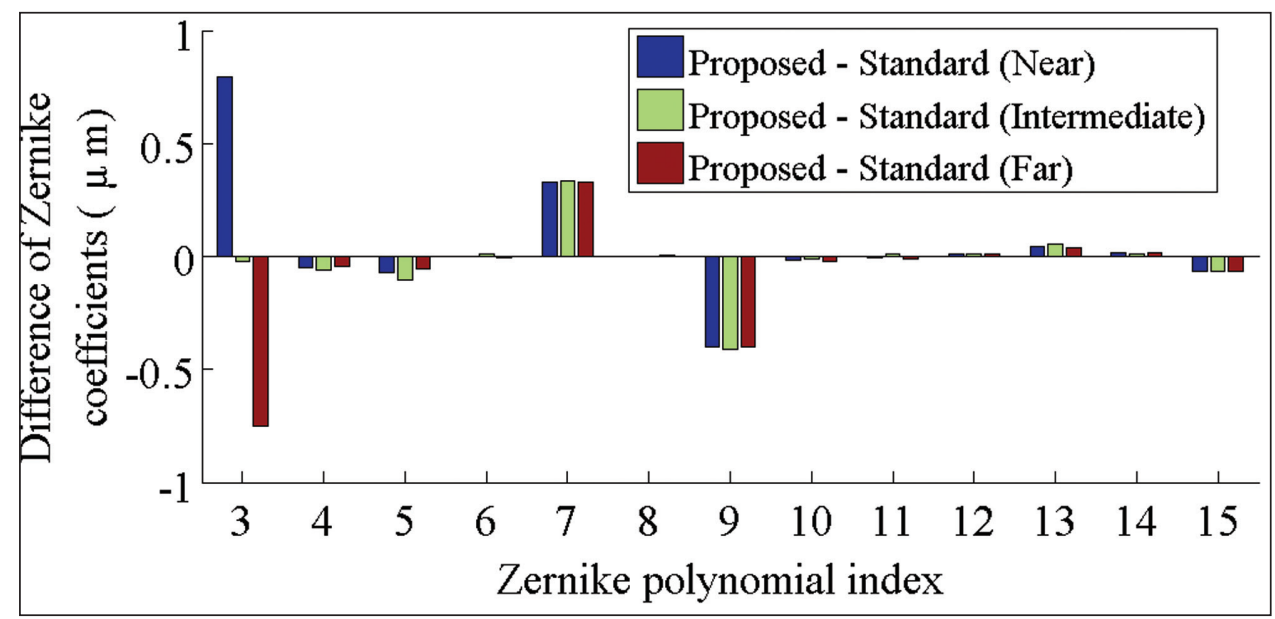

Figure 3. Difference between the reconstructed higher order Zernike coefficients (in the customized computer eye model of Figure 6) obtained with the proposed method and the reconstructed Zernike coefficients obtained with the standard reconstruction method in a single plane of focus at near (blue bars), intermediate (green bars), or far (brown bars) distances. Data are for a 4-mm pupil diameter.

matched the corneal HOA predicted with OCT-based wavefront analysis (Figure CA). The residual wavefront obtained by subtracting the corneal aberration map shown in Figure CA and the wavefront reconstructed using the proposed multifocal reconstruction method (Figure CE) was found to have a dominant defocus term $(0.55 \mu \mathrm{m})$. The reconstructed wavefront corresponds to the pupil plane and the aberrations are induced by the cornea, which is separated by a fixed distance from the pupil plane, justifying the presence of defocus in this residual wavefront error.

The reconstructed wavefronts shown in Figures CBCE are decomposed using Zernike polynomials. The differences between the Zernike coefficients predicted with the standard reconstruction method (using fixed single plane measurements at near, intermediate, and far distances) and the proposed multifocal reconstruction method are shown in Figure 3. Vertical coma (approximately $0.33 \mu \mathrm{m}$ ) and trefoil terms (approximately $-0.40 \mu \mathrm{m}$ ) dominate the differences between the two methods, similar to Figure 2.

\section{WAVEFRONT RECONSTRUCTION FROM LRT ABERROMETRY}

Figure 4A shows the phase due to the Lentis Mplus IOL calculated with ray tracing simulations. The yellow colored circles inscribed with a red cross show the locations of LRT sampling. The standard wavefront reconstruction method was applied to far fixation (Figure 4B) and near fixation (Figure 4C) slope measurements in a real patient. Figure 4D shows the wavefront reconstructed with the proposed multifocal reconstruction method that combines the slope values at far and near distances. The absolute magnitude of primary vertical coma coefficient alone with measurements made in a single plane (intermediate fixation) is $0.69 \mu \mathrm{m}$. With the proposed multifocal reconstruction method that combines the wavefront slopes, the absolute magnitude of primary vertical coma was reduced to $0.15 \mu \mathrm{m}$. A com- parison of the standard and proposed methods in terms of the detected coma-like, trefoil-like, and other HOAs is shown in Figure 4E.

\section{DISCUSSION}

A major objective of the development of multifocal IOLs is to provide spectacle independence to patients. Segmented refractive multifocal IOLs with a rotationally asymmetric surface embedded near section have proven to provide comfortable far and near functional vision. ${ }^{2}$ In addition, this design allows the reduction of the sources of scattering, halos, and glare. ${ }^{21}$ Aberrometry is an important clinical tool for objective diagnosis and eye examination. Here, it is shown that the direct application of conventional and commercial wavefront sensors is not appropriate for accurate estimation of the aberrations in patients implanted with the rotationally asymmetric refractive multifocal IOLs. This is due to the coupling of the phase due to the power pupillary distribution of the multifocal IOL and the true aberrations of the patient. It becomes even more significant when abrupt changes in multifocal IOL design are present, which is the case with most segmented designs. To our knowledge, this is the first attempt to evaluate the true HOA decoupled from the effects of the power distribution due to the multifocal component of an IOL. The proposed multifocal reconstruction method, which combines the local wavefront slope measurements at far and near fixation conditions, is applicable to any instrument that uses the local wavefront slopes to evaluate the aberrations and hence would be applicable to the LRT instrument used in this study and most other commercial devices based on the principles of the Hartmann-Shack wavefront sensor.

In comparison with the simple eye model case, there is an increase in the magnitude of the spherical aberration of the reconstructed wavefront in the 


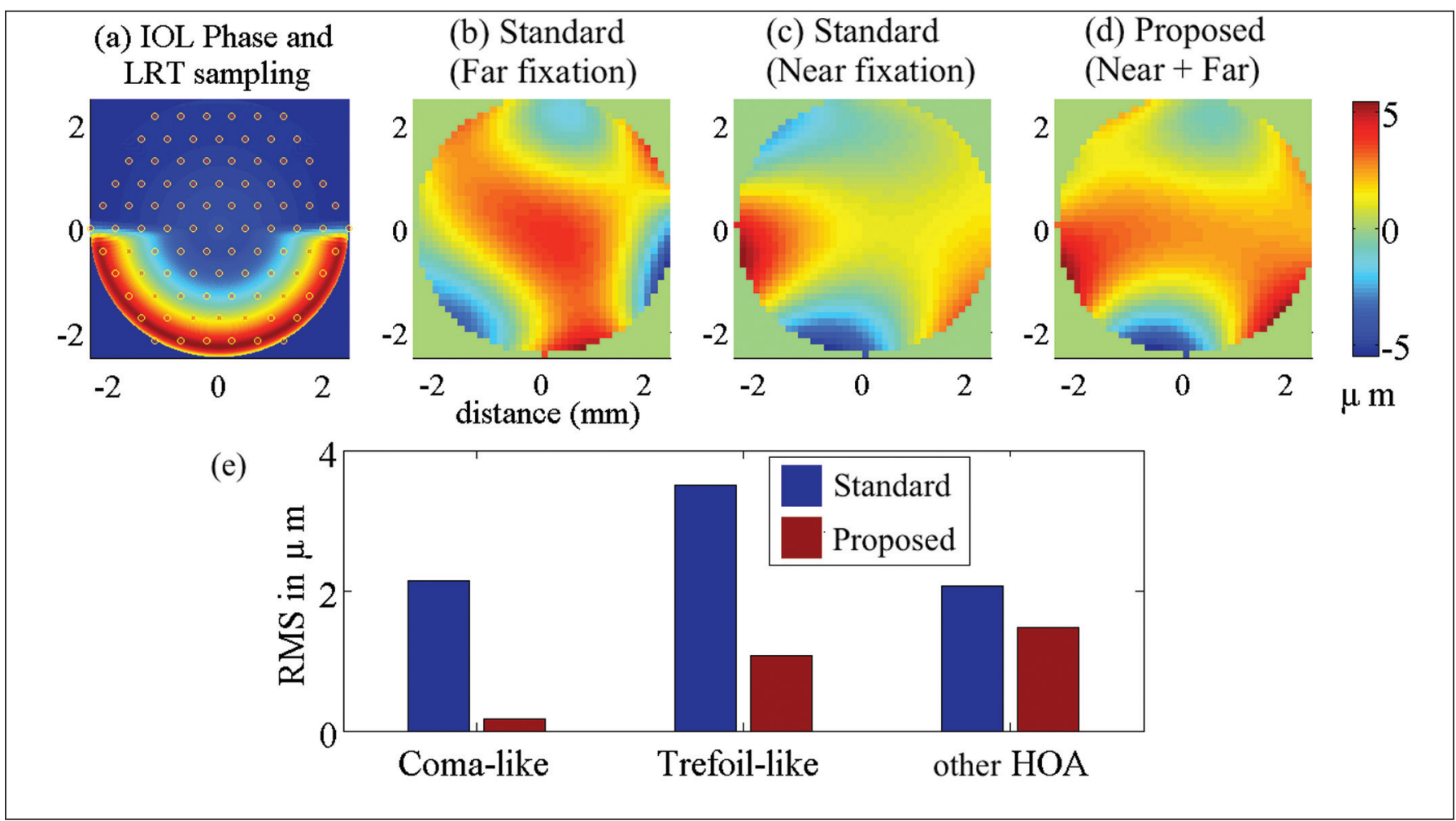

Figure 4. (A) Phase due to the Lentis Mplus intraocular lens (IOL) (Oculentis $\mathrm{GmbH}$, Berlin, Germany) calculated with ray tracing simulations in a pseudophakic computer eye model. The yellow colored circles inscribed with a red cross show the 91 locations of laser ray tracing (LRT) sampling on a hexagonal grid. Wavefronts reconstructed from LRT measurements in a real patient implanted with the Lentis Mplus IOL using the standard reconstruction method at a single plane: (B) far and (C) near. (D) Wavefront reconstructed using the proposed multifocal reconstruction method that combines the slope measurements at far and near fixation distances. (E) Coma-like, trefoil-like, and other higher order aberrations (HOA) obtained with the standard method (single plane-intermediate fixation) and the proposed method. Data are for a 5-mm pupil diameter.

case of the pseudophakic computer eye model (Figure AD), which was found to be partly due to the transition region, placement of the IOL in the eye, and the axial length difference between the two eye models (with the crystalline lens and the multifocal IOL). The Zernike coefficient of the primary vertical coma predicted with the standard reconstruction method ( 0.34 $\mu \mathrm{m}$ ) in this study is comparable to the difference of the mean coma-like RMS between a monofocal group and a multifocal group implanted with the Lentis Mplus IOL reported in the literature. ${ }^{4}$ It should be noted that this value depends on the near addition specification of the multifocal IOL. Although the wavefront aberrations are different, the magnitudes of vertical coma and trefoil are consistent in Figures 2-3. This shows the effectiveness of the new approach in separating the true ocular HOA and to reliably describe the phase map in eyes implanted with multifocal IOLs as a combination of HOA and the IOL power map. This true ocular HOA map differs from that obtained from a standard wavefront reconstruction method, where the reconstructed wave aberration is typically dominated by vertical primary coma aberration and trefoil.
In the case of intermediate fixation, a large magnitude of tilt, which arises due to the partial defocus in near and far correcting portions of the IOL with opposite signs (an implication of which is shown in the primary defocus coefficients of Figure 4), was subtracted to indicate the strong presence of coma in the wavefront reconstructed using the standard reconstruction method and zonal fitting (see reconstructed wavefront in Figure 2C).

From Figure 4A, it can be seen that there are a few slope measurements that are made near the transition region. These measurements can be sources of error in wavefront reconstruction. The jump in phase near the transition region can cause errors in wavefront estimation because the spots coming from the transition region are not adequately focused in near or far fixation and hence result in a few questionable slope measurements. An approach to deal with this problem is to avoid using the slope data coming from the transition region. In practice, it is difficult to identify the transition region and determine the slopes arising due to it. An alternate approach that was used here is to apply the proposed multifocal reconstruction method wherein the transition region is assumed to be in either 

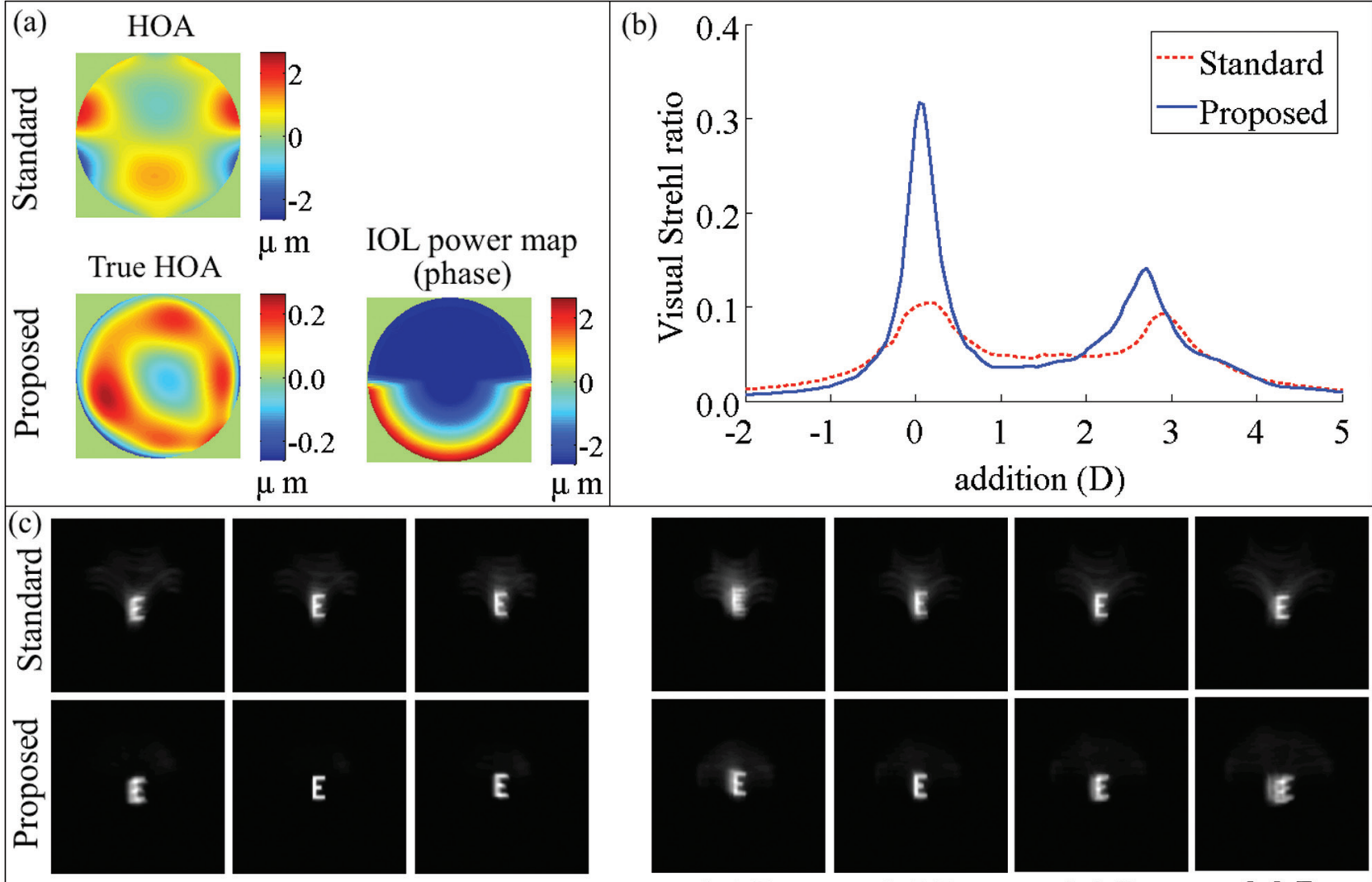

$-0.3 \mathrm{D}$

$0.0 \mathrm{D}$

$0.3 \mathrm{D}$

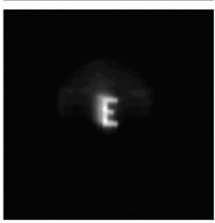

$2.4 \mathrm{D}$

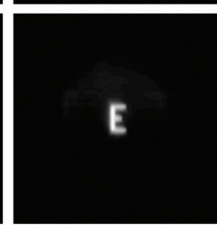

2.7 D

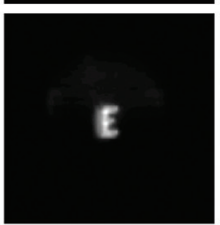

3.0 D

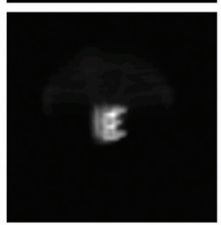

$3.3 \mathrm{D}$

Figure 5. (A) Proposed method disentangles the higher order aberrations (HOA) from the intraocular lens (IOL) power distribution, whereas the standard reconstruction method that uses single plane measurements leads to a large magnitude primary vertical coma. (B) Estimated through-focus visual Strehl ratio in a computer eye model with Lentis Mplus IOL (Oculentis GmbH, Berlin, Germany), using the wavefront aberrations derived from the application of standard reconstruction method (red dotted line), or the proposed multifocal reconstruction method (blue line). (C) Simulated through-focus images as predicted by the proposed method in comparison with the standard method. Calculations are for a 4-mm pupil diameter. D = diopters

near or far correcting zones. The effects of the transition region can then be suppressed by decomposing the reconstructed wavefront using a sufficient number of Zernike polynomials (Figure AD). It may be noted that a direct application of the modal fitting approach on the slope measurements after appropriately combining near and far fixation slope measurements will also help in nullifying the errors due to the transition region.

The proposed multifocal reconstruction method disentangles the true HOA from the phase map due to the power distribution of the multifocal IOL also in a customized computer eye model for a patient, which is not achievable with the standard reconstruction method as illustrated in Figure 5A. The difference between the true HOA detected with the proposed method and the HOA detected with the standard method is dominantly primary vertical coma and trefoil. This study also adds to the knowledge of the performance of ro- tationally asymmetric IOLs, particularly while understanding that the primary vertical coma detected using standard aberrometry does not contribute to expanding the depth of focus. Indeed, it is found that throughfocus visual Strehl ratio estimated using the aberrometry analysis with the standard reconstruction method (ie, dominated by primary vertical coma) or that arising from an analysis of the eye's aberrations and the phase map produced by the IOL with the proposed multifocal reconstruction method differ dramatically (Figure 5B). Although the true wavefront analysis produces a distinct simultaneous vision through-focus optical quality curve consistent with the bifocal design of the Lentis Mplus IOL, ${ }^{6}$ the standard reconstruction analysis that results in a coma-dominated wavefront does not reproduce the expected through-focus curve, further supporting the fact that a coma-dominated pattern poorly represents the actual wave aberration in patients implanted with the Lentis Mplus IOL. Analy- 
sis based on through-focus simulated images (Figure 5C, with letter ' $E$ ') showed that the proposed method allows a better estimate of the true capabilities of a rotationally asymmetric segmented multifocal IOL (Lentis Mplus) from aberrometry data.

The proposed multifocal reconstruction method can be potentially applied to other multifocal IOL designs. It is straightforward to apply the method to other angularly segmented refractive designs ${ }^{22,23}$ and to radially segmented IOLs, provided that the wavefront slope sampling is sufficiently high to map each zone.

A new method of extracting the true HOA separately to the phase map produced by the power pupillary distribution of the IOL in patients implanted with rotationally asymmetric multifocal IOLs has been proposed. The method, which uses aberrometry measurements at multiple planes, was tested through simulations on an OCT-based customized eye model and validated on data from an LRT aberrometer. The results show that the previously reported occurrence of a large magnitude primary vertical coma in patients implanted with the Lentis Mplus IOL is due to inadequate analysis of the wavefront sensor measurements.

\section{AUTHOR CONTRIBUTIONS}

Study concept and design (VA, CD, SM); data collection (VA, PP-M, IJ-A); analysis and interpretation of data (VA, PP-M, EM-E, CD, NA, $S M)$; writing the manuscript (VA, SM); critical revision of the manuscript (VA, PP-M, EM-E, CD, NA, IJ-A, SM); statistical expertise (VA); administrative, technical, or material support (CD, SM); supervision $(C D, S M)$

\section{REFERENCES}

1. Artigas JM, Menezo JL, Peris C, Felipe A, Diaz-Llopis M. Image quality with multifocal intraocular lenses and the effect of pupil size: comparison of refractive and hybrid refractive-diffractive designs. J Cataract Refract Surg. 2007;33:2111-2117.

2. McAlinden C, Moore JE. Multifocal intraocular lens with a surface-embedded near section: short-term clinical outcomes. J Cataract Refract Surg. 2011;37:441-445.

3. Ramón ML, Piñero DP, Pérez-Cambrodí RJ. Correlation of visual performance with quality of life and intraocular aberrometric profile in patients implanted with rotationally asymmetric multifocal IOLs. J Refract Surg. 2012;28:93-99.

4. Alió JL, Piñero DP, Plaza-Puche AB, Chan MJR. Visual outcomes and optical performance of a monofocal intraocular lens and a new-generation multifocal intraocular lens. J Cataract Refract Surg. 2011;37:241-250.

5. Gatinel D. Limited accuracy of Hartmann-Shack wavefront sensing in eyes with diffractive multifocal IOLs. J Cataract Refract Surg. 2008;34:528.
6. Radhakrishnan A, Dorronsoro C, Marcos S. Differences in visual quality with orientation of a rotationally asymmetric bifocal intraocular lens design. J Cataract Refract Surg. 2016;42:12761287.

7. Platt BC, Shack R. History and principles of Shack-Hartmann wavefront sensing. J Refract Surg. 2001;17:S573-S577.

8. Moreno-Barriuso E, Lloves JM, Marcos S, Navarro R, Llorente L, Barbero S. Ocular aberrations before and after myopic corneal refractive surgery: LASIK-induced changes measured with laser ray tracing. Invest Ophthalmol Vis Sci. 2001;42:1396-1403.

9. Moreno-Barriuso E, Marcos S, Navarro R, Burns SA. Comparing laser ray tracing, the spatially resolved refractometer and the Hartmann-Shack sensor to measure the ocular wave aberration. Optom Vis Sci. 2001;78:152-156.

10. Llorente L, Diaz-Santana L, Lara-Saucedo D, Marcos S. Aberrations of the human eye in visible and near infrared illumination. Optom Vis Sci. 2003;80:26-35.

11. Liou H, Brennan NA. Anatomically accurate, finite model eye for optical modeling. J Opt Soc Am A. 1997;14:1684-1695.

12. Southwell WH. Wave-front estimation from wave-front slope measurements. J Opt Soc Am. 1980;70:998-1006.

13. Akondi V, Falldorf C, Marcos S, Vohnsen B. Phase unwrapping with a virtual Hartmann-Shack wavefront sensor. Opt Express. 2015;23:25425-25439.

14. Schwiegerling J. Statistical generation of normal and post-refractive surgery wavefronts. Clin Exp Optom. 2009;92:223-226.

15. Ortiz S, Pérez-Merino P, Durán S, et al. Full OCT anterior segment biometry: an application in cataract surgery. Biomed Opt Express. 2013;4:387-396.

16. Ortiz S, Siedlecki D, Grulkowski I, et al. Optical distortion correction in optical coherence tomography for quantitative ocular anterior segment by three-dimensional imaging. Opt Express. 2010;18:2782-2796.

17. Ortiz S, Siedlecki D, Remon L, Marcos S. Optical coherence tomography for quantitative surface topography. Appl Opt. 2009;48:6708-6715.

18. Pérez-Merino P, Velasco-Ocana M, Martinez-Enriquez E, Marcos S. OCT-based crystalline lens topography in accommodating eyes. Biomed Opt Express. 2015;6:5039-5059.

19. Sun M, Pérez-Merino P, Martinez-Enriquez E, Velasco-Ocana M, Marcos S. Full 3-D OCT-based pseudophakic custom computer eye model. Biomed Opt Express. 2016;7:1074-1088.

20. Llorente L, Marcos S, Dorronsoro C, Burns SA. Effect of sampling on real ocular aberration measurements. J Opt Soc Am A. 2007;24:2783-2796.

21. Venter JA, Pelouskova M, Collins BM, Schallhorn SC, Hannan SJ. Visual outcomes and patient satisfaction in 9366 eyes using a refractive segmented multifocal intraocular lens. J Cataract Refract Surg. 2013;39:1477-1484.

22. de Gracia P, Dorronsoro C, Marcos S. Multiple zone multifocal phase designs. Opt Lett. 2013;38:3526-3529.

23. Vinas M, Dorronsoro C, Gonzalez V, Cortes D, Radhakrishnan A, Marcos S. Testing vision with angular and radial multifocal designs using adaptive optics. Vis Res. In press. 


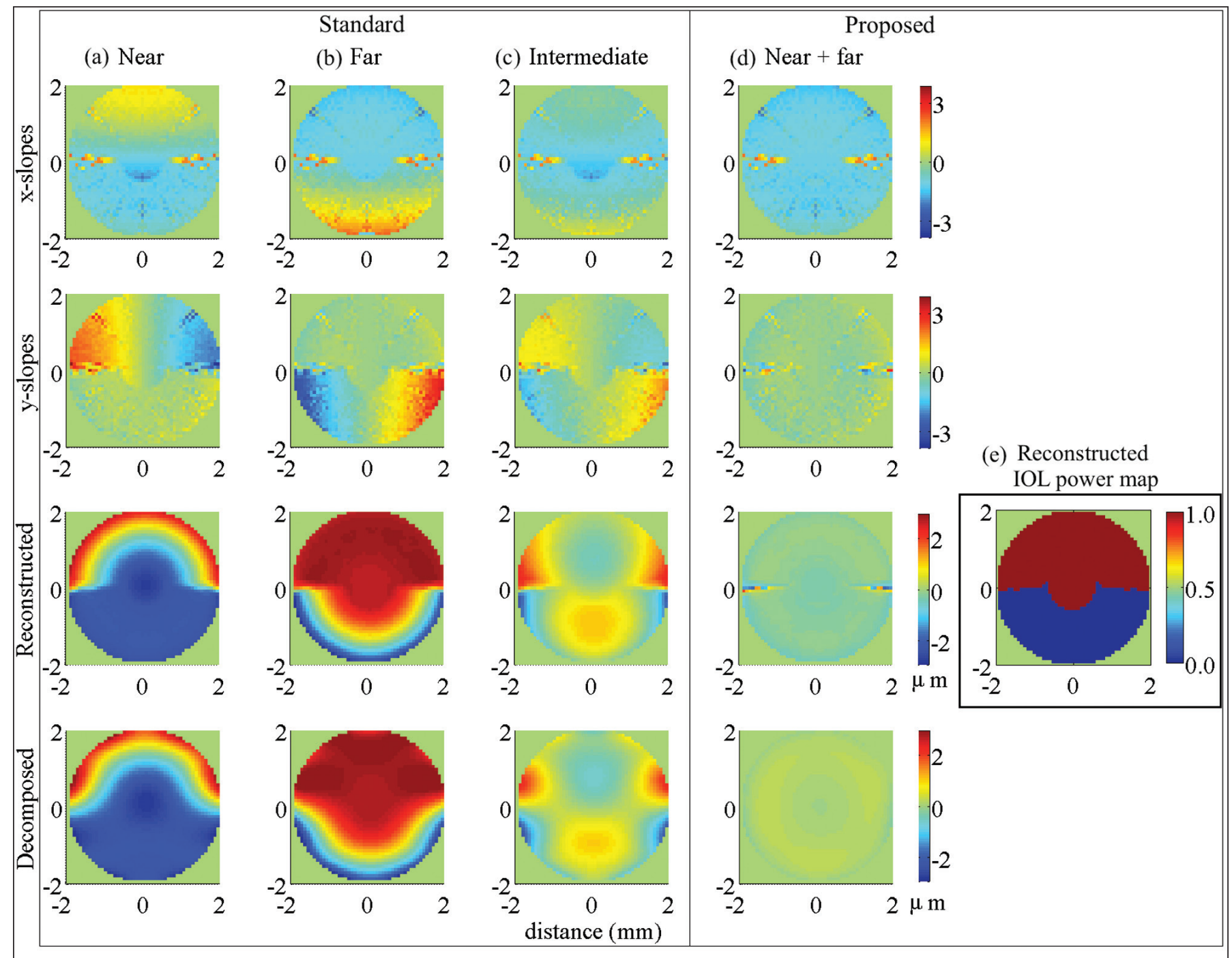

Figure A. Simulated local ' $x$ ' and ' $y$ ' slopes, the reconstructed and decomposed wavefronts obtained using the standard reconstruction method at (A) near, (B) far, and (C) intermediate distances in a pseudophakic computer eye model with the Lentis Mplus intraocular lens (IOL) (Oculentis GmbH, Berlin, Germany) and with no aberrations. (D) True local ' $x$ ' and ' $y$ ' slopes, the true reconstructed wavefront and decomposed wavefront obtained with the proposed multifocal reconstruction method. (E) The binary multifocal IOL power map reconstructed with the proposed method ('O' for the near and ' 1 ' for the far correcting portion of the multifocal IOL). 


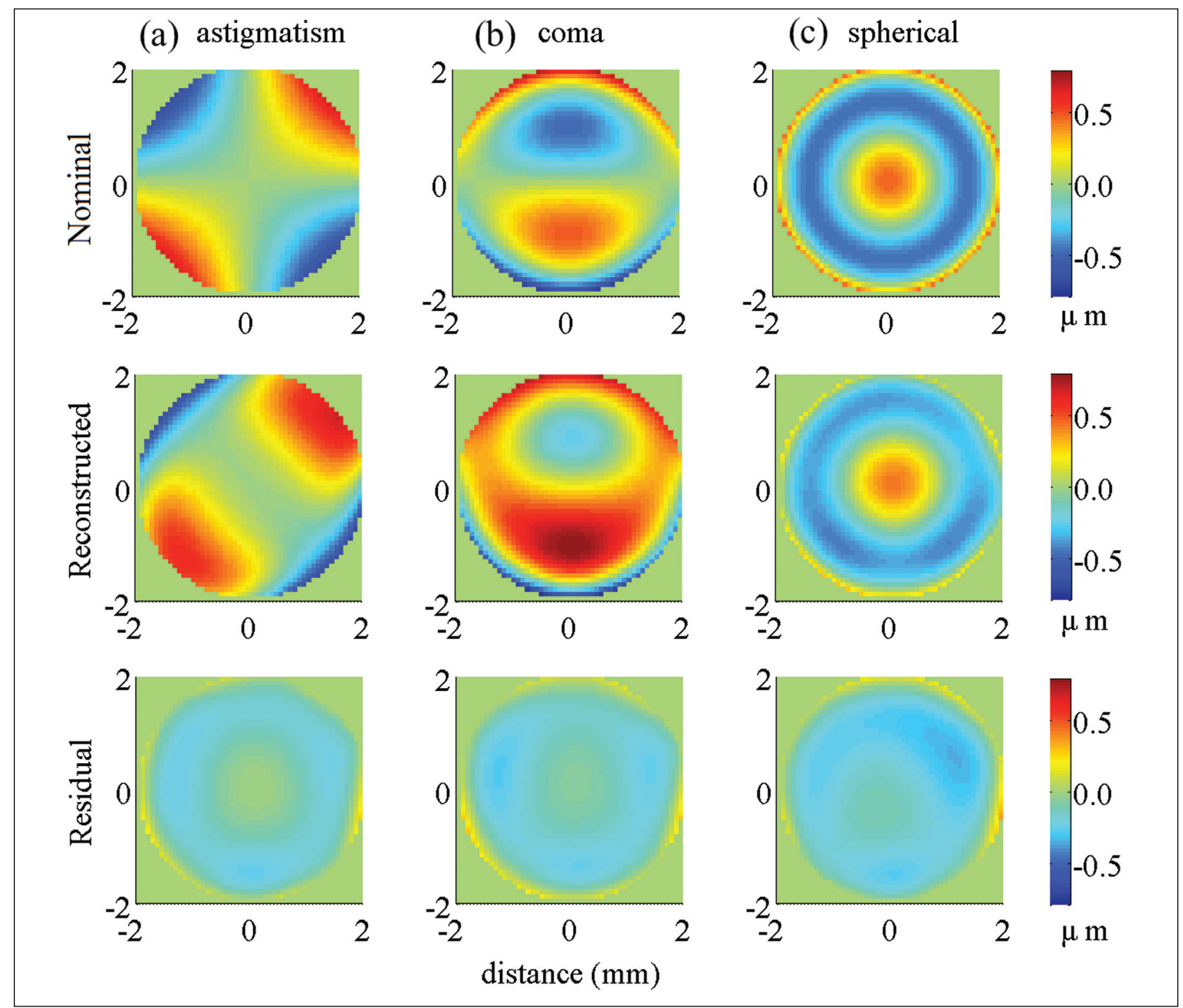

Figure B. Comparison of nominal wavefronts and reconstructed wavefronts obtained with the proposed multifocal reconstruction method in pseudophakic computer eye models implanted with the Lentis Mplus intraocular lens (IOL) (Oculentis GmbH, Berlin, Germany), decoupled from the IOL phase map, in eyes with nominal (A) primary $45^{\circ}$ astigmatism, (B) vertical primary coma, and (C) primary spherical aberrations. 


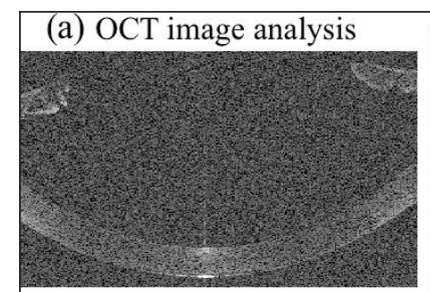

(i) Anterior chamber depth $3.684 \pm 0.003 \mathrm{~mm}$

(ii) Corneal thickness $0.541 \pm 0.001 \mathrm{~mm}$

(iii) Radius of curvature (anterior) $7.73 \pm 0.07 \mathrm{~mm}$

(iv) Radius of curvature (posterior) $6.48 \pm 0.09 \mathrm{~mm}$

Corneal aberration map

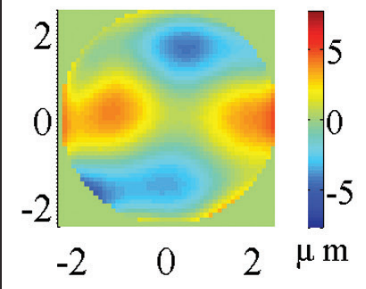

(b) Near
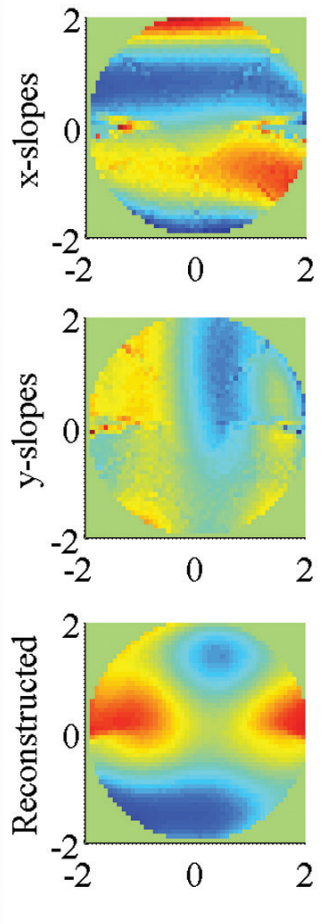

(c) Far
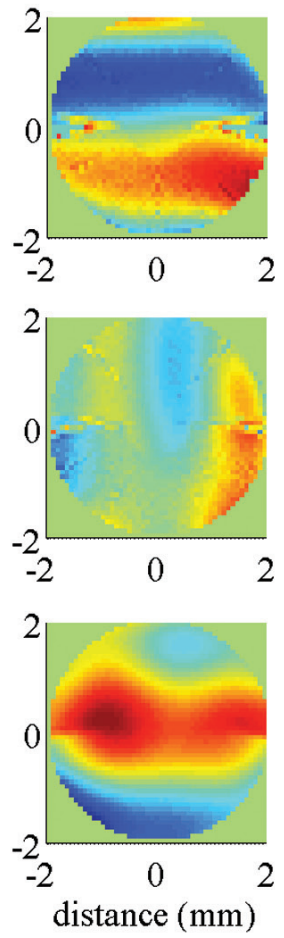

(d) Intermediate
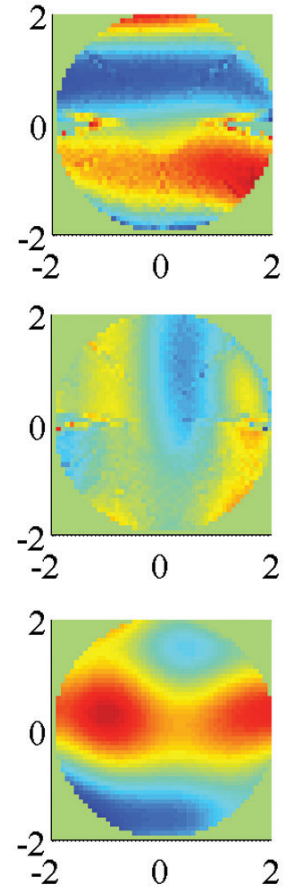

(e) Near + far
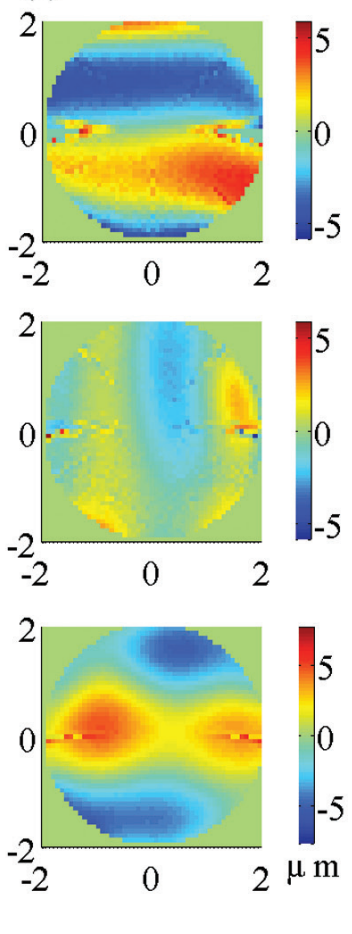

Figure C. (A) Two-dimensional cross-sectional image, anterior segment biometry, and corneal aberrations derived from quantitative custom optical coherence tomography (OCT)-based measurements in a patient. Simulated local ' $x$ ' and ' $y$ ' slopes and wavefronts obtained using standard reconstruction method at (B) near, (C) far, and (D) intermediate distances in a customized computer eye model based on OCT measurements. (E) True local ' $x$ ' and ' $y$ ' slopes and the wavefront reconstructed with the proposed multifocal reconstruction method. 